\title{
Voir pour payer: ou comment transformer le système de soins et l'acte médical en une vaste entreprise de Peep-show
}

Demander aux médecins que leur pratique soit efficace, adéquate et économique n'a, en soi, rien de choquant puisque la médecine a toujours cherché et pratiqué dans ce sens. Par contre, la volonté d'inscrire ces principes dans une loi (art. 32, LAMal) est plutôt singulière: à ma connaissance, aucun autre groupe professionnel n'est soumis aussi explicitement à une telle réglementation. Cela n'est pas sans conséquences sur l'ensemble du système.

Cette différence de traitement me place face à une question: la nature de cet article de loi voulu par le législateur vise-t-elle réellement une supposée non-qualité de notre pratique médicale ou au contraire, n'a-t-elle pas davantage une visée répressive latente qui tente de répondre à l'obsession (ou la paranoïa?) des assureurs dans la traque de supposés abus de pratique? Des abus de bien sociaux qu'ils feraient d'ailleurs mieux de nous exposer concrètement, plutôt que de créer un climat général délétère de suspicion qui nuit à la qualité de nos relations avec eux, ainsi qu'à celles entretenues avec les patients.

Pour le corps médical, le risque consiste à donner consistance et à nourrir cette suspicion d'abus généralisé logée dans le fantasme de nos partenaires. C'est pourquoi je souhaite que le corps médical se garde de se lancer dans des réponses précipitées et univoques de légitimation (ou de déculpabilisation?) comme, par exemple, la course effrénée et aveugle vers l'affichage externe d'une médecine fondée sur les preuves (ou l'evidencebased medicine). En raison de la spécificité (nonunivocité de la symptomatologie) et de la complexité du raisonnement clinique qui procède par élimination d'hypothèses, la définition de la qualité dans les pratiques médicales est une affaire interne à la médecine. Nous devons rester attentifs à ce que nos propres recherches sur nos processus de soins et de pratiques ne soient pas - à l'extérieur perverties en «normes de non-abus» économiques de soins. Une information plus régulière de ce qu'est la pratique médicale me semble indispensable mais rendre compte ne signifie pas rendre des comptes. Le risque de cette course folle est de nourrir la logique du Peep-show: faites-nous voir et nous payerons. Avec en prime, pour l'assureur et plus grave pour les patients aussi, l'illusion que, s'ils ont $\mathrm{vu}$, ils savent.

Depuis l'introduction du TARMED, la pratique médicale a donné beaucoup à voir; peu de professions libérales ont accepté de se déshabiller à ce point. Cela dit, malgré les inconvénients, le résultat n'est pas négatif car le corps médical a aussi pu comprendre ce qu'il pouvait tirer de cet effeuillage vis-à-vis de ses partenaires politiques et économiques: la possession et l'analyse de ses propres chiffres. Une conscience de son propre corps, de ses organes et évidemment du désir de l'autre. Nos chiffres ont ainsi permis de rendre les interprétations et les déclarations intempestives de nos interlocuteurs beaucoup plus difficiles. C'est une bonne chose! Néanmoins, il n'est pas possible de continuer à construire un système de soins ni des relations thérapeutiques et financières dans un tel contexte de méfiance réciproque.

Cette pression, entretenue par l'illusion que voir c'est savoir, est fortement soutenue par les assureurs. Elle est relayée sans aucun esprit critique par une classe politique, ce qui pousse la collectivité et les patients vers des demandes toujours plus irréalistes et pressantes: avoir immédiatement le bon examen qui fera tomber le bon diagnostic et le bon traitement ... en un minimum de temps et de frais! Toute durée supplémentaire prise dans cette logique d'immédiateté est aujourd'hui suspecte de corruption. Le corps médical n'est évidemment pas opposé à montrer ce qu'il fait, mais cette logique témoigne d'une insistante et d'une profonde méconnaissance de la pratique médicale, de la science et de l'art du diagnostic ainsi que de la thérapeutique; tous procèdent par hypothèses, par élimination et surtout par un serrage progressif de la clinique pour aboutir enfin à un diagnostic différentiel et des propositions de traitement. Selon mon appréciation, c'est bien ce temps de comprendre que l'on ne veut plus payer.

A l'avenir, le corps médical va donc donner encore davantage à voir, mais ce que les assureurs vont voir n'est probablement pas ce qu'ils désirent voir. Non pas qu'il y ait une résistance du côté médical, mais parce que, comme dans le Peep-show, ce qu'ils désirent réellement voir ne se voit pas!

Quant à la tâche qui incombe au monde politique, je lui adresserais volontiers ce slogan trouvé dans une de mes récentes lectures: «Sur terre en tout cas, les politiques ne méritent leur nom que s'ils combattent l'esprit de domestication.» [1]

Dr René Raggenbass, membre du Comité central de la FMH

1 Milner J-C. La politique des choses. Paris: Navarin; 2005. 


\section{Erst wird gezeigt, dann bezahlt - oder wie das Gesundheitswesen und die medizinische Praxis zu einem riesigen Peepshow-Geschäft werden}

Dass man von den Ärztinnen und Ärzten bei der Ausübung ihrer Tätigkeit Wirksamkeit, Zweckmässigkeit und Wirtschaftlichkeit verlangt, ist an sich keineswegs schockierend, weil die medizinische Forschung und Praxis schon immer dieses Ziel verfolgt haben. Diese Prinzipien in einem Gesetz (Art. $32 \mathrm{KVG}$ ) verankern zu wollen, ist jedoch eher eigenartig. Meines Wissens gibt es keine andere Berufsgruppe, die so explizit einer derartigen Reglementierung untersteht. Dies bleibt jedoch nicht ohne Folgen für das Gesamtsystem.

Diese Sonderbehandlung wirft für mich folgende Frage auf: Bezweckt diese vom Gesetzgeber gewollte Bestimmung wirklich die Behebung eines angeblichen Qualitätsmangels in der Medizin, oder dient sie nicht im Gegenteil einer latenten Repression, mit der versucht wird, eine Antwort auf die Obsession (oder Paranoia?) der Versicherer bei der Jagd nach angeblichen Missbräuchen zu geben? Die Versicherer würden im übrigen besser daran tun, uns solche Missbräuche zulasten der Allgemeinheit konkret aufzuzeigen, statt ein allgemeines Verdachtsklima zu schaffen, das unseren Beziehungen nicht nur zu ihnen, sondern auch unseren Patientinnen und Patienten schadet.

Für die Ärzteschaft besteht die Gefahr, dass dieser Verdacht auf verbreitete Missbräuche, der in der Phantasie unserer Partner verwurzelt zu sein scheint, gefestigt und genährt wird. Deshalb würde ich mir wünschen, dass sich die Ärzteschaft vor übereilten und eindeutigen Legitimierungs- oder Entlastungsreaktionen hüte. Ein Beispiel dafür ist das ungezügelte und blinde Propagieren einer nachweisgestützten Medizin (Evidence-based Medicine). Der klinische Ansatz, der darin besteht, Hypothesen aufzustellen und mittels Ausschlussverfahren wieder $\mathrm{zu}$ verwerfen, weist besondere Eigenheiten auf (Uneinheitlichkeit der Symptomatologie) und ist komplex aufgebaut. Folglich ist die Definition von Qualität in der medizinischen Praxis eine interne Angelegenheit der Medizin. Wir müssen aufpassen, dass unsere eigene Forschung über unsere Pflegeverfahren und -praxis nicht draussen $\mathrm{zu}$ «Missbrauchsverhinderungsnormen» pervertiert wird. Regelmässigere Informationen über die medizinische Praxis scheinen mir unverzichtbar, aber «informieren» bedeutet nicht «Rechenschaft ablegen». Dieser verrückte Wettlauf birgt die Gefahr, dass wir einer Peepshow-Logik verfallen: Zeigt uns etwas, und wir bezahlen. Zudem erliegen die Versicherer und - noch schlimmer - die Patientinnen und Patienten der Illusion «ich habe gesehen, also weiss ich».
Seit der Einführung des TARMED hat die Medizin viel offengelegt. Es gibt nur wenige freie Berufe, die bereit waren, sich so sehr zu entblössen. Trotz der Nachteile ist jedoch das Ergebnis nicht negativ, weil die Ärzteschaft auch begriffen hat, was sie von diesem Striptease vor den Partnern aus Politik und Wirtschaft erhält: die Verfügbarkeit und Analyse der eigenen Zahlen und ein Bewusstsein über den eigenen Körper mit seinen Organen - und natürlich die Sehnsucht des anderen. Unsere Zahlen haben unseren Gesprächspartnern ihre vorschnellen Interpretationen und Erklärungen erschwert. Und das ist doch eine gute Sache! Trotzdem können wir in einem solchen, von gegenseitigem Misstrauen geprägten Umfeld nicht weiter am Aufbau eines Pflegesystems sowie von therapeutischen und finanziellen Beziehungen arbeiten.

Der Druck der Illusion, wonach Sehen auch Wissen bedeutet, wird von den Versicherern kräftig verstärkt und von der Politik völlig kritiklos weitergegeben. Dies treibt die Gesellschaft und die Patienten zu immer unrealistischeren und drängenderen Forderungen: sofort die richtige Untersuchung mit der richtigen Diagnose und der richtigen Behandlung, und das Ganze mit möglichst wenig Zeitaufwand und Kosten! In dieser Logik der Unmittelbarkeit erweckt jede zusätzlich beanspruchte Zeit den Verdacht der Korruption. Die Ärzteschaft ist natürlich nicht dagegen, zu zeigen, was sie tut. Die obenbeschriebene Vorstellung zeugt jedoch von einer hartnäckigen und tiefverwurzelten Verkennung der medizinischen Praxis, der Wissenschaft, Diagnostik und Therapeutik. Alle nutzen Hypothesen, Ausschlussverfahren und vor allem eine schrittweise Eingrenzung des klinischen Bildes, um schliesslich zu einer differenzierten Diagnose und zu entsprechenden Behandlungsvorschlägen $\mathrm{zu}$ gelangen. Mir scheint, als wolle man genau diese zum Verstehen notwendige Zeit nicht mehr bezahlen.

Die Ärzteschaft wird folglich in Zukunft noch mehr zeigen müssen, aber die Versicherer werden wohl nicht das zu sehen bekommen, was sie wollen. Der Grund ist aber nicht der Widerstand der Ärzte, sondern der Grundsatz der Peepshow: Was sie wirklich sehen wollen, kann man gar nicht sehen!

In bezug auf die von der Politik zu lösende Aufgabe möchte ich den Verantwortlichen folgenden Satz mitgeben, den ich in meiner jüngsten Lektüre gefunden habe: «Sur terre en tout cas, les politiques ne méritent leur nom que s'ils combattent l'esprit de domestication» [1].

Dr. med. René Raggenbass, Mitglied des Zentralvorstandes FMH gegen den Zähmungsgeist kämp- Milner J-C. La politique choses. Paris: Navarin; 2005.
1 Freie Übersetzung: Auf der Erde jedenfalls haben Politiker ihren Namen nur verdient, wenn sie 